\title{
A Case Report of Monomorphic Epithelial Intestinal T-Cell Lymphoma and Literature Review
}

\author{
Jing Deng1, Yongguang Zou1*, Guangshu $\mathrm{Li}^{2}$ \\ ${ }^{1}$ Department of Cardiology, Xiaogan Hospital, Wuhan University of Science and Technology, Xiaogan, China \\ ${ }^{2}$ Department of Gastroenterology, Xiaogan Hospital, Wuhan University of Science and Technology, Xiaogan, China \\ Email:605959406@qq.com, *3283974019@qq.com, lgs2006@163.com
}

How to cite this paper: Deng, J., Zou, Y.G. and Li, G.S. (2020) A Case Report of Monomorphic Epithelial Intestinal T-Cell Lymphoma and Literature Review. International Journal of Clinical Medicine, 11, 15-22.

https://doi.org/10.4236/ijcm.2020.112002

Received: January 13, 2020

Accepted: February 9, 2020

Published: February 12, 2020

Copyright $\odot 2020$ by author(s) and Scientific Research Publishing Inc. This work is licensed under the Creative Commons Attribution International License (CC BY 4.0).

http://creativecommons.org/licenses/by/4.0/

\begin{abstract}
Background: Monomorphic epithelial intestinal T-cell lymphoma (MEITL), previously known as type II Enteropathy-associated T-cell lymphoma (EATL), is a rare intestinal tumor with strong invasiveness, poor prognosis, atypical clinical manifestations, and is difficult to diagnose. Aim: The clinical manifestations, imaging, histopathology, and immunohistochemical characteristics, treatment and prognosis of this case of MEITL were analyzed retrospectively, and combined with literatures learning to provide the experiences and lessons for diagnosis and treatment. Case Presentation: Here, we present a 68-year-old Asian woman with unexplained intestinal perforation and lung imaging changes as the first manifestation, and gradually appearing refractory diarrhea and pulmonary cavities. During the period, due to the unknown diagnosis, all treatments were mainly based on anti-infection and antidiarrhea, but her symptoms had no improvement after therapy, even died of poor basic physical conditions. Colonoscopy biopsy showed atypical lymphocyte infiltration-like growth and the formation of local superficial ulcers. Immunohistochemical tests showed that T-lymphocyte hyperplasia was predominant and led to a diagnosis of MEITL. Conclusion: MEITL is mainly manifested by gastrointestinal symptoms and lacks specific symptoms. The diagnosis of MEITL should be based on comprehensive judgment of clinical manifestations, pathological features and immunohistochemical detection results. Positive biopsy confirmed and timely chemotherapy may be conductive to a better prognosis.
\end{abstract}

\section{Keywords}

MEITL, Pulmonary Cavities, Refractory Diarrhea, Intestinal Perforation 


\section{Introduction}

EATL is a rare intestinal intraepithelial T-cell lymphoma, which is a subtype of non-Hodgkin's lymphoma. It consists of two types, Type I (classic) EATL, composed of large tumor cells and typically manifested as celiac disease, accounts for $80 \%-90 \%$ of all cases, which were mainly distributed in European countries. The other type of EATL, composed of small to medium-sized tumor cells, was mainly distributed in Asian countries, which accounted for 10\% - 20\% [1]. In the newly revised WHO lymphoma typing of 2016 [2], type II EATL was renamed as MEITL. Some international reports pointed out that affected parts of MEITL are mainly involved in the abdominal cavity and intra-abdominal lymph nodes with gastrointestinal symptoms such as diarrhea as the main manifestation, occasionally found in the skin, ovary, chest and other extraintestinal organs; however there were no reports of combined lung changes as the initial performance. Here, a special MEITL case is presented with the permission of the patient, the clinical manifestations, pathological features, diagnosis and treatment process was summarized and discussed.

\section{Case Report}

A 68-year-old woman was diagnosed as "small intestine perforation" due to "abdominal pain" on May 20, 2018. Then emergency surgery was performed, but the reason of perforation was not found out during the operation. Then the chest CT showed "pulmonary infection with pleural effusion" during hospitalization, but no signs of infection. The symptoms were improved by giving anti-infective treatment and the patient was discharged. After re-examination, pleural effusion disappeared, but "pulmonary infection lesions" still existed and gradually expanded to form multiple cavities. The patient was hospitalized again because of coughing for half a year, exacerbating for three months on August 8, 2018. During that period, two bronchoscopy biopsy showed inflammatory changes and one ultrasound fiber optic plexus biopsy showed multiple lymphocytes. The result of PET/CT examination combined with medical history was considered to be neoplastic lesions, but no histopathological evidence, except anti-tumor treatment, other treatments were performed for the patient such as anti-infective (Levofloxacin, Piperacillin tazobactam), relieving cough and reducing sputum, but the symptoms were not obviously improved and sputum with blood streaks was occasionally found, then the magnetic navigation puncture and surgery were recommended to diagnosed, but the relatives of patient refused, and the conservative treatment was discharged after more than 20 days. During the hospitalization, the patient has diarrhea 2 - 3 times a day, and montmorillonite powder was given for treatment. After discharge, the diarrhea gradually increased to more than 10 times a day with yellow bloating, accompanied by abdominal pain, poor appetite, vomiting, and weight loss. Then the patient came back to hospital again on September 25, 2018.

After hospitalization, chest CT (Figure 1(a), Figure 1(b)) showed multiple 
cavities, nodules and solid changes in the lungs. These symptoms were recommended to identify chronic infection and tumor metastasis. Abdominal CT enhancement (Figure 1(c), Figure 1(d)) showed rectal and sigmoid edema, which could be potentially inflammatory. Colonoscopy (Figure 2(a), Figure 2(b)) showed irregular shallow ulcers with different sizes were distributed in the end of ileum, ascending colon, descending colon, and sigmoid colon, respectively. Pathology (Figure 3(a)): the infiltrating growth of lymphoid cells with small volume in the mucosal epithelium was observed and infiltrated into the lamina propria. Morphology and immunohistochemistry were consistent with monomorphic epithelial intestinal T-cell lymphoma (MEITL). In situ hybridization of tumor cells: EBER (-). Immunohistochemistry shows (Figures 3(b)-(f)): LCA (+), CD3 (+), CD2 (+), CD7 (+), CD43 (+), CD5 (-), CD56 (+), Perforin (-), Granzyme B (-), CD20 (-), CD79a (-), CD4 (-), CD8 (-), CD138 (-), CD30 $(-)$, CD34 (-), VIM (+), pCK (-), Ki -67 (LI: 50\%).

Laboratory examination results: the patient had no obvious blood abnormalities in the blood routine in the past six months, but mild anemia, malnutrition and electrolyte imbalance occurred in the late stage due to long-term intractable diarrhea. Liver and kidney function, coagulation function, rotavirus detection, thyroid function, a complete set of lupus, pANCA, cANCA, PPD test, stool culture, IgA, IgG, IgM, complement C3, RF, anti-CCP antibody, ASO, T-SPOT and clostridium difficile culture of stool were normal. Complement C4 $0.38 \mathrm{~g} / \mathrm{L} \uparrow$;

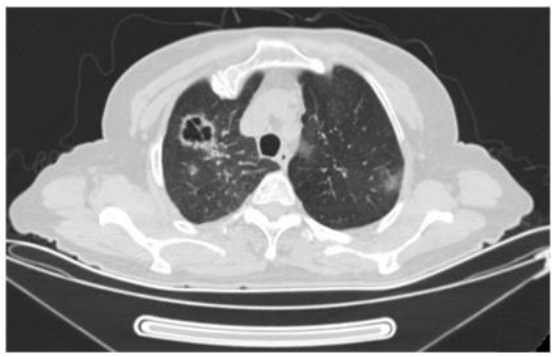

(a)

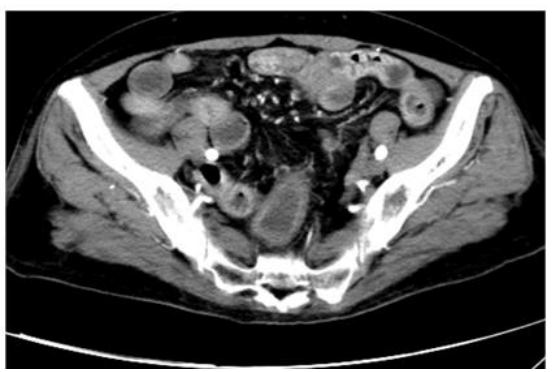

(c)

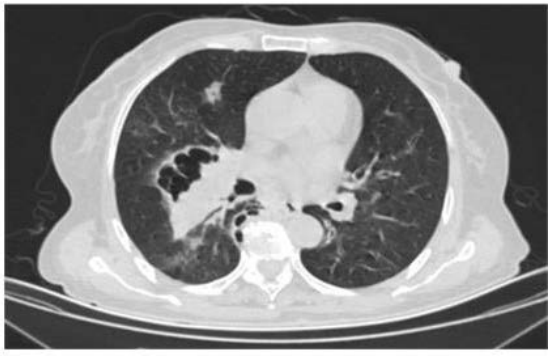

(b)

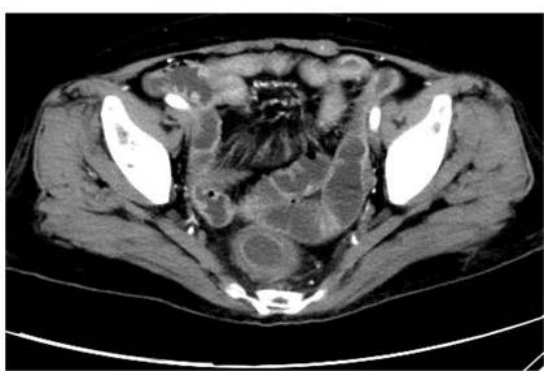

(d)

Figure 1. (a) (b) CT scan of the chest in multiple sites showed multiple cavities in the lungs and exhibited a honeycomb change. Multiple nodules were obvious in the lungs and some of them exhibited glass-like changes; (c) (d) Abdominal CT enhancement showed that thickness of the rectum and sigmoid colon wall increased, enhanced mucosal was mildly intensified and exhibited concentric ring-like changes, peripheral fat gap became blurred. 


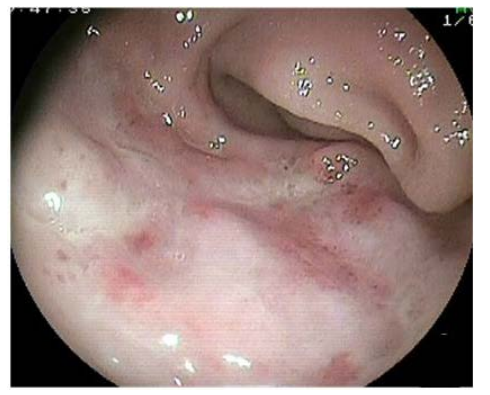

(a)

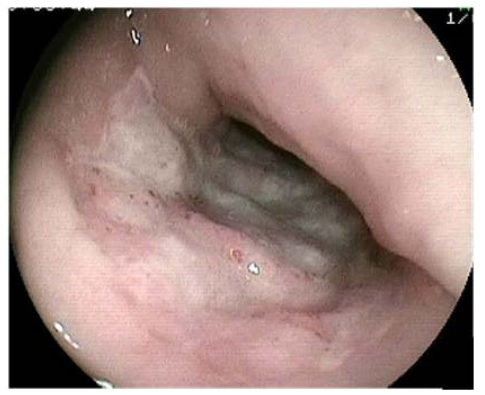

(b)

Figure 2. Multiple irregular shallow ulcers can be observed in many parts of the large intestine under colonoscopy with clear borders, covered with white moss, soft texture. The granulation tissue changes at the bottom of the sigmoid colon.

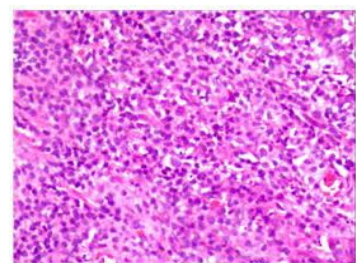

(a)

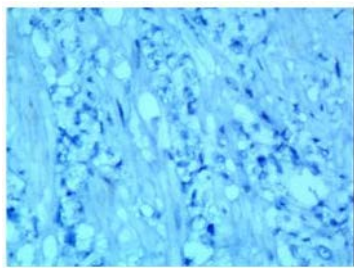

(d)

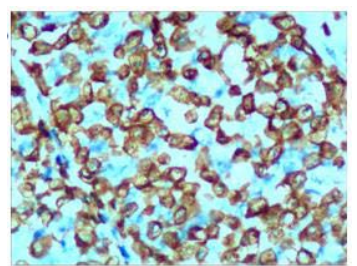

(b)

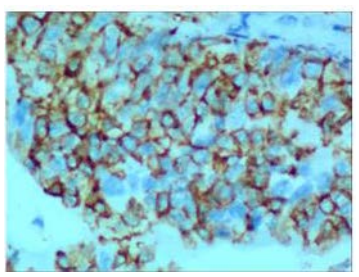

(e)

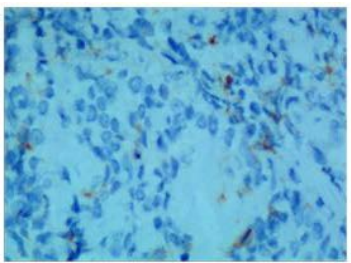

(c)

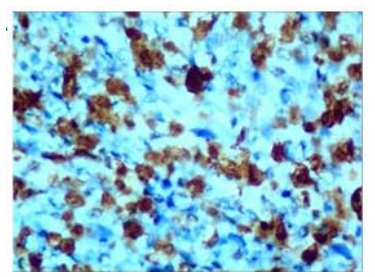

(f)

Figure 3. Immunohistochemistry observations of MEITL tissue ((a): HE`200); Tumor cells showed positive for CD3 (b), negative for CD8 (c), CD30 (d), CD56 (e), Ki-67 index of $50 \%$ (f).

C-12 tumor marker: CA199 37.27 IU/mL $\uparrow$; CA125 35.63 IU/mL $\uparrow$; Cyfra21-1 $8.70 \mathrm{ng} / \mathrm{ml} \uparrow$; PCT $0.26 \mathrm{ng} / \mathrm{ml}$; ESR $21.00 \mathrm{~mm} / \mathrm{h} \uparrow$, CRP $55.70 \mathrm{mg} / \mathrm{L} \uparrow$. Sputum culture: Enterobacter aerogenes.

During the hospitalization, the patients were given symptomatic treatments such as antidiarrheal (Montmorillonite), anti-infection (Cefoperazone), stomach protection (Pantoprazole) and nutritional rehydration, but the condition was getting worse, even with a dramatic loss of weight. Because of poor effect of chemotherapy, extremely easy access to intestinal perforation and poor prognosis, the patient and his families refused to chemotherapy and discharged. One month later, the patient died.

\section{Discussion}

It is difficult to diagnose MEITL because of the extremely low morbidity, lacking of characteristic symptoms and endoscopic features. The case we presented here is combined lung changes and intestine perforation as the initial performance, 
without considering these two symptoms as a manifestation of one disease, which caused misdiagnosis and missed diagnosis. It's finally confirmed until half-year data of the patient was collected for consultation and the pathological specimens with special staining were sent to the pathology department of the superior hospital.

\subsection{Clinical Manifestation}

MEITL is mainly found in middle-aged and elderly men, with a male to female ratio of about 6:1, mainly involved in the abdominal cavity and intra-abdominal lymph nodes with gastrointestinal symptoms such as diarrhea as the main manifestation, more common in the small intestine, followed by the large intestine and stomach. In combination with previous reports of such cases at home and abroad, the disease has various symptoms such as skin damage caused by skin infiltration [3], ascites, ovarian mass [4], pleural effusion [5], central nervous system [6], autoimmune hemolytic anemia [7], etc. Other non-specific manifestations include abdominal pain, diarrhea, abdominal mass, constipation, etc. systemic manifestations of anorexia, weight loss, etc. which was often misdiagnosed as infectious enteritis, inflammatory bowel disease and intestinal tuberculosis that can explain the patient's chronic diarrhea with obvious symptoms of consumption [8] [9]. Sometimes, intestinal perforation and obstruction are the first manifestations, thus we should pay attention to identify such common diseases. In this case, pulmonary cavity changes are considered to be the most likely metastasis of MEITL in the lungs, with metastasis in the early stage. However, due to the low incidence of this disease, three fiberoptic bronchoscopic biopsies did not perform special staining, so no diagnostic results were found, but combined with imaging findings, pulmonary cavities manifested in immunocompromised patients may result from bacterial infection, aspergillosis, tuberculosis or non-tuberculous mycobacterial etc. [10]. So it's not ruled out that this case is MEITL combined with other diseases. The disease progressed rapidly and the prognosis was poor, the onset to the final diagnosis was 5 months and the survival period was 6 months while the median survival time was only 11 months in previous reported [11]. The main causes of death of this disease are malnutrition, infection, systemic failure, etc.

\subsection{Pathology and Endoscopic Features}

The diagnosis of the disease mainly relies on pathological examination. Some patients are diagnosed due to "acute abdomen" undergoing surgical resection of the intestinal segment biopsy, other patients depend on endoscopic biopsy. Commonly used endoscopic biopsies include enteroscopy, gastroscopy, and colonoscopy. A small portion of cases makes a definite diagnosis by a biopsy of extraintestinal organs and tissues. Enteroscopy is an expensive and invasive procedure, and difficult to operate, which is not the preferred endoscopy for disease diagnosis. In the early endoscope stage of MEITL, the main manifestations are ulcers of different depths, with no special forms, which caused most of 
them were misdiagnosed as Crohn's disease or intestinal tuberculosis. The middle and late ulcers deteriorate into penetration and develop into large and deep ulcers, where the surface is uneven, the bottom is covered with white moss and around the ulcer as insect eroding, normal mucosa could be observed between the ulcers, and intestinal tube with ulcers are obviously edema and thickened, but generally no intestinal stenos is [12]. The pathogenesis of MEITL may be related to glucan enteropathy and EB virus infection but the specific mechanism is unknown [13]. In this case, EBER (-), but human anti-glutenin antibodies (AGA) could not be determined due to lack of technology. MEITL is a rare type of lymphoma that originates from intestinal T lymphocyte. Immunohistochemically, tumor cells are CD3+, CD5-, CD4-, CD8+, CD56+, CD30-, and EBER[14].

\subsection{Treatment}

There is currently no standardized treatment. Some scholars believe that surgery can remove lesions, reduce tumor burden, avoid bleeding and perforation, but the rate of curative resection is low and postoperative complications are common. Another part of the reports suggests that surgery combined with chemotherapy or chemotherapy alone would be the first choice. The most commonly used chemotherapy for MEITL in previous reports of domestic and foreign is anthracyclines and CHOP [15] or R-CHOP (rituximab, cyclophosphamide, doxorubicin, vincristine, prednisone) [14]. A few reports have tried other therapy such as gemcitabine instead of anthracyclines [14], hyper-CVAD (cyclophosphamide + doxorubicin + vincristine + dexamethasone/methotrexate + cytarabine) [4] and Polyethylene glycol combined with asparaginase [16], etc. Some patients have a survival period that exceeds the expected median survival, but the efficacy remains to be confirmed by more cases. In this case, the patient is mainly treated symptomatically, chemotherapy is recommended after pathological diagnosis, but treatment is abandoned because of poor prognosis. Currently all treatments for MEITL patients show low survival rate, which require more research to find out innovative therapies, such as transplantation, targeted drug and autologous stem cell transplantation after high-dose chemotherapy may be a better option for MEITL treatment [17].

\section{Conclusion}

In summary, because of the low incidence and difficulty to distinguish from Crohn disease, intestinal tuberculosis and other types of lymphoma of the gastrointestinal tract, MEITL is hard to diagnose and treat in time. It invaded and progressed rapidly, with poor response to current treatment, resulting in a bad prognosis. During the diagnosis, it should be highly suspected to be MEITL if the patient has unexplained refractory diarrhea, pulmonary cavities, intestinal perforation, no efficacy after long-term conservative treatment and is combined with multiple ulcers of the intestine and systemic symptoms. Then active biopsy should be combined with immunohistochemistry, and the results are recom- 
mended to obtain a comprehensive judgment to avoid missed diagnosis or misdiagnosis.

\section{Conflicts of Interest}

The authors declare no conflicts of interest regarding the publication of this paper.

\section{References}

[1] Song, M.J., Park, C.S., Hwang, H.S., et al. (2014) A Case of Type II Enteropathy-Associated T-Cell Lymphoma with Epstein-Barr Virus Positivity. Korean Journal of Pathology, 48, 426. https://doi.org/10.4132/KoreanJPathol.2014.48.6.426

[2] Sakhdari, A. and Medeiros, L.J. (2019) Update on Lymphoma Classification: The 2016 Revised World Health Organization Classification of Hematopoietic and Lymphoid Neoplasms. Advances in Cell and Gene Therapy, 2, e57. https://doi.org/10.1002/acg2.57

[3] Aiempanakit, K., Amatawet, C., Chiratikarnwong, K., et al. (2017) Erythema Multiforme-Like Cutaneous Lesions in Monomorphic Epitheliotropic Intestinal T-Cell Lymphoma: A Rare Case Report. Journal of Cutaneous Pathology, 44, 183-188. https://doi.org/10.1111/cup.12864

[4] Wang, L., Huang, D.H., Xiao, C.Y., et al. (2018) Monomorphic Epitheliotropic Intestinal T-Cell Lymphoma: A Clinic-Pathologic Study of 3 Cases and Literature Review. Medical Journal of Chinese People's Liberation Army, 43, 45-50.

[5] Antoniadou, F., Dimitrakopoulou, A., Voutsinas, P.M., et al. (2017) Monomorphic Epitheliotropic Intestinal T-Cell Lymphoma in Pleural Effusion: A Case Report. Diagnostic Cytopathology, 45, 1050-1054. https://doi.org/10.1002/dc.23772

[6] Kubota, Y. and Kusaba, K. (2018) Monomorphic Epitheliotropic Intestinal T-Cell Lymphoma Involving the Central Nervous System. Blood, 131, 1765-1765. https://doi.org/10.1182/blood-2017-11-819136

[7] Kato, A., Takiuchi, Y., Aoki, K., et al. (2011) Enteropathy-Associated T-Cell Lymphoma Type II Complicated by Autoimmune Hemolytic Anemia. Journal of Clinical and Experimental Hematopathology, 51, 119-123.

https://doi.org/10.3960/jslrt.51.119

[8] Samuel, R., Krill, T. and Merwat, S. (2019) Monomorphic Epitheliotropic Intestinal T-Cell Lymphoma: A Rare Cause of Chronic Diarrhea. Journal of Gastrointestinal Cancer, 50, 1051-1054. https://doi.org/10.1007/s12029-019-00210-3

[9] Jiao, G.H., Zheng, Z.Q. and Jiang, K. (2014) Enteropathy-Associated T-Cell Lymphoma Presenting with Gastrointestinal Tract Symptoms: A Report of Two Cases and Review of Diagnostic Challenges and Clinicopathological Correlation. Oncology Letters, 8, 91-94. https://doi.org/10.3892/ol.2014.2105

[10] Gadkowski, L.B. and Stout, J.E. (2008) Cavitary Pulmonary Disease. Clinical Microbiology Reviews, 21, 305-333. https://doi.org/10.1128/CMR.00060-07

[11] Roberti, A., Dobay, M.P., Bisig, B., et al. (2016) Type II Enteropathy-Associated T-Cell Lymphoma Features a Unique Genomic Profile with Highly Recurrent SETD2 Alterations. Nature Communications, 7, Article No. 12602. https://doi.org/10.1038/ncomms12602

[12] Wang, Y.N., Li, J., Ni, Y.H., et al. (2018) The Clinical Characteristics of Patients with Monomorphic Epitheliotropic Intestinal T-Cell Lymphoma Characterized by Minor Endoscopic Abnormalities. Chinese Journal of Internal Medicine, 57, 
112-117.

[13] Wang, L., Chen, Y., Pan, X.Y., et al. (2011) Application of Ig/TCR Gene Rearrangement Analysis Combined with in Situ Hybridization with EBER in the Diagnosis of Gastrointestinal Lymphomas. Journal of Clinical \& Experimental Pathology, 27, 580-585.

[14] Hong, Y.S., Woo, Y.S., Park, G., et al. (2016) Endoscopic Findings of Enteropathy-Associated T-Cell Lymphoma Type II: A Case Series. Gut Liver, 10, 147-151. https://doi.org/10.5009/gnl14457

[15] Novakovic, B.J., Novakovic, S. and Frkovic-Grazio, S. (2006) A Single-Center Report on Clinical Features and Treatment Response in Patients with Intestinal T Cell Non-Hodgkin's Lymphomas. Oncology Letters, 16, 191-195.

https://doi.org/10.3892/or.16.1.191

[16] Gentille, C., Qin, Q., Barbieri, A., et al. (2017) Use of PEG-Asparaginase in Monomorphic Epitheliotropic Intestinal T-Cell Lymphoma, a Disease with Diagnostic and Therapeutic Challenges. Ecancer, 11, 771.

https://doi.org/10.3332/ecancer.2017.771

[17] Bishton, M.J. and Haynes, A.P. (2007) Combination Chemotherapy Followed by Autologoustem Cell Transplant for Enteropathy-Associated T Cell Lymphoma. British Journal of Haematology, 136, 111-113.

https://doi.org/10.1111/j.1365-2141.2006.06371.x 